\title{
The Cluster Magnetic Field Investigation: overview of in-flight performance and initial results
}

\author{
A. Balogh ${ }^{1}$, C. M. Carr ${ }^{1}$, M. H. Acuña ${ }^{2}$, M. W. Dunlop ${ }^{1}$, T. J. Beek ${ }^{1}$, P. Brown ${ }^{1}$, K.-H. Fornacon ${ }^{3}$, E. Georgescu ${ }^{4}$, \\ K.-H. Glassmeier ${ }^{3}$, J. Harris ${ }^{1}$, G. Musmann ${ }^{3}$, T. Oddy ${ }^{1}$, and K. Schwingenschuh ${ }^{5}$ \\ ${ }^{1}$ Imperial College, London, UK \\ ${ }^{2}$ NASA/GSFC, Greenbelt, Md., USA \\ ${ }^{3}$ IGM, TU Braunschweig, Germany \\ ${ }^{4} \mathrm{MPE}$, Garching, Germany \\ ${ }^{5}$ IfW, Graz, Austria
}

Received: 21 March 2001 - Revised: 18 June 2001 - Accepted: 19 June 2001

\begin{abstract}
The accurate measurement of the magnetic field along the orbits of the four Cluster spacecraft is a primary objective of the mission. The magnetic field is a key constituent of the plasma in and around the magnetosphere, and it plays an active role in all physical processes that define the structure and dynamics of magnetospheric phenomena on all scales. With the four-point measurements on Cluster, it has become possible to study the three-dimensional aspects of space plasma phenomena on scales commeasurable with the size of the spacecraft constellation, and to distinguish temporal and spatial dependences of small-scale processes. We present an overview of the instrumentation used to measure the magnetic field on the four Cluster spacecraft and an overview the performance of the operational modes used in flight. We also report on the results of the preliminary in-orbit calibration of the magnetometers; these results show that all components of the magnetic field are measured with an accuracy approaching $0.1 \mathrm{nT}$. Further data analysis is expected to bring an even more accurate determination of the calibration parameters. Several examples of the capabilities of the investigation are presented from the commissioning phase of the mission, and from the different regions visited by the spacecraft to date: the tail current sheet, the dusk side magnetopause and magnetosheath, the bow shock and the cusp. We also describe the data processing flow and the implementation of data distribution to other Cluster investigations and to the scientific community in general.
\end{abstract}

Key words. Interplanetary physics (instruments and techniques) - magnetospheric physics (magnetospheric configuration and dynamics) - space plasma physics (shock waves)

\section{Introduction: scientific objectives of the Cluster Mag- netic Field Investigation}

Magnetic fields are a key component of space plasmas. They provide the basic physical reference system for the transport

Correspondence to: A. Balogh (a.balogh@ic.ac.uk) of momentum and energy in the plasma. Boundaries between plasmas of different origin, which often consist of thin current sheets, are recognised primarily through their signatures in magnetic field observations. Wave phenomena in the plasma are also ordered with respect to the underlying magnetic field. The Cluster mission brings a further, unexplored dimension to magnetic field observations: using the measurement of the magnetic field vector at four points, a range of new parameters, such as the current density vector, the propagation vectors of waves, as well as the orientation, non-planarity, and motion of boundary surfaces can be determined. Additionally, the four-point magnetic field measurements are needed for the determination of the internal structure of the boundary regions. Such measurements are also essential for describing the parameters of turbulence in the different plasma regimes in and around the Earth's magnetosphere. Magnetic field vector measurements at the four Cluster spacecraft provide, therefore, an essential ingredient for meeting the scientific objectives of the mission.

The primary objective of the Cluster Magnetic Field Investigation (FGM) is to provide accurate measurements of the magnetic field vector at the location of the four Cluster spacecraft. The many scientific objectives that are related to the physics of space plasmas in the Earth's magnetosphere and in its vicinity all use the basic measurements of the magnetic field vector. We enumerate here a brief summary of the detailed objectives presented previously (Balogh et al., 1993, 1997).

The Cluster orbit samples most of the key regions of the magnetosphere. In the dayside boundary of the magnetosphere, both at mid-latitudes and in the cusp, processes associated with magnetic reconnection and turbulence are believed to occur which control the dynamics of the solar wind interaction with the Earth's magnetic field (e.g. Paschmann, 1995; Scholer, 1995). On the nightside, in the near-Earth magnetospheric tail, frequent large-scale magnetic reconfigurations occur during substorms in which the tail plasma and current sheets plays a key role. On the flanks and on the dayside of the magnetosphere, there is a wealth of plasma 


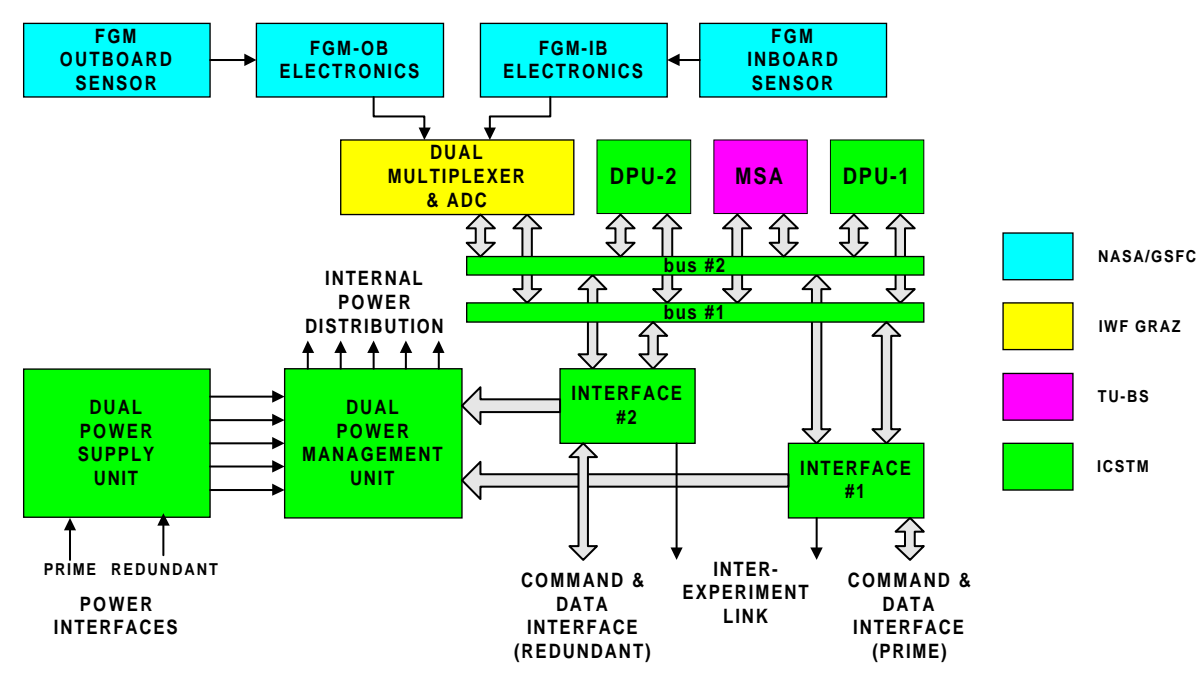

Fig. 1. Block diagram of the FGM instrument on Cluster.

phenomena on many scales at the bow shock and in the magnetosheath (for a first four-point study of mirror structures in the magnetosheath, see Lucek et al., 2001). The anisotropy which is inherent in all magnetised plasma processes, introduced by the magnetic field, makes the accurate determination of the magnetic field at high time resolution a critical contribution to the probing of small-scale structures and dynamics encountered in these regions (Southwood, 1990).

The current understanding of the structure and dynamics of the main boundaries on the sunward side of the magnetosphere, the bow shock and the magnetopause is based, to a large extent, on observations of single crossings and numerical simulations. Some two-point observations are available that have identified some of the temporal and spatial variability at these boundaries and in the upstream medium (Thomsen, 1988; Elphic, 1988 and Russell, 1988). However, these boundaries have complex, evolving structures and are non-stationary on several time- and length scales. Fourpoint magnetic field observations provide information on the immediate neighbourhood of the boundaries that clarify the phenomenology of boundary-associated phenomena simultaneously at the four locations. Magnetopause structure is clearly a complex topic, with relevance to a wide range of phenomena that contribute (and are affected by) the detailed processes that occur at this boundary. The first magnetic field investigation of the magnetopause by Cluster has been presented by Dunlop et al. (2001a); a joint investigation of the magnetopause using low frequency wave and magnetic field data has been carried out by Rezeau et al. (2001).

A particularly interesting objective of Cluster on this topic is the exploration of processes occuring at quasi-parallel bow shock geometries, where the generally accepted shock reformation occurs (Scholer and Burgess, 1992). Boundary crossing phenomena include complex wave fields that are an integral part of the MHD processes that form the wider environment of the boundaries; inherent non-stationarity prevents the resolution of these wave fields by single- or even two-point measurements. During a two-year mission, Cluster will cross the dayside bow shock and magnetopause several hundred times. This will allow the observation of both boundaries under a range of conditions that provide the basis for a separation of different phenomena and causal processes, as outlined in numerical simulations before the mission (e.g. Giacalone et al., 1994). The magnetometers on Cluster have already shown that they can generate the necessary data sets to determine the context and processes at these boundaries (see Sect. 6 and Horbury et al., 2001).

Other specific phenomena for four-point analysis to which the observations of the magnetic field on Cluster will contribute include dayside magnetic reconnection and the nature of flux transfer events in particular; the role of the KelvinHelmholtz instability in magnetopause processes; impulsive events, possibly driven by discontinuities in the solar wind; and, as a prime objective of Cluster, the nature, structure and dynamics of the outer cusp. For a first exploration of the capabilities of the Cluster magnetic field observations in the cusp, see Cargill et al. (2001).

In the magnetospheric tail, Cluster observations will target both phenomena at small-scales, such as the structure and time evolution of current sheets associated with the growth phase of substorms and, on larger scales, to establish the magnetic topology of the different regions in the tail and their dynamics. The role, in particular, of a range of current structures and their magnetic signatures, all on scales which require high time resolution magnetic measurements, simultaneously at several locations, will contribute to resolving the time evolution of the magnetospheric tail prior to and during substorms.

A significant subset of the objectives of the magnetic field investigation has close connections with the wider field of solar-terrestrial relations. In this context, collaboration with simultaneous ground-based observations is already playing a key role in relating magnetospheric responses with signatures observed by remote-sensing, ground-based instruments 
Table 1. Operative ranges for the FGM (not all used)

\begin{tabular}{ccc}
\hline Range No. & Range & Resolution \\
\hline 2 & $-64 \mathrm{nT}$ to $+63.97 \mathrm{nT}$ & $7.8 \times 10^{-3} \mathrm{nT}$ \\
3 & $-256 \mathrm{nT}$ to $+255.87 \mathrm{nT}$ & $3.1 \times 10^{-2} \mathrm{nT}$ \\
4 & $-1024 \mathrm{nT}$ to $+1023.5 \mathrm{nT}$ & $0.125 \mathrm{nT}$ \\
5 & $-4096 \mathrm{nT}$ to $+4094 \mathrm{nT}$ & $0.5 \mathrm{nT}$ \\
7 & $-65536 \mathrm{nT}$ to $+65504 \mathrm{nT}$ & $8 \mathrm{nT}$ \\
\hline
\end{tabular}

and facilities, such as magnetometer chains and ionospheric radars (Wild et al., 2001).

The analysis of four-point magnetic field measurements presents formidable conceptual and technical challenges. The techniques developed for Cluster have a rich literature; an important review of the multipoint analysis techniques was published by a Working Group of the International Space Science Institute (Paschmann and Daly, 1998, chapters and references therein). The Cluster magnetometer team also published several aspects of four-point analysis techniques specifically related to magnetic field data since 1990 (for a comprehensive list of publications on this topic, see Balogh and Dunlop, 2000, but also Dunlop et al., 1996, 1997; Dunlop and Woodward, 1998, 2000). First reviews of applications of these techniques using Cluster magnetic field data are presented in Dunlop et al. (2001b), and Glassmeier et al. (2001).

\section{Overview of the FGM instrument}

The FGM instrument on each spacecraft consists of two triaxial fluxgate magnetic field sensors on one of the two radial booms of the spacecraft, and an electronics unit on the main equipment platform. The block diagram of the instrument is shown in Fig. 1. The instrument is designed to be highly failure-tolerant through a full redundancy of all its functions. In particular, either of the two magnetometer sensors can be used as the primary sensor for the main data stream from the instrument, although in normal operations, the outboard sensor, located at the end of the $5 \mathrm{~m}$ radial boom, is designated as the primary source of the data. The magnetometers can measure the three components of the field in seven ranges, although only four of these (numbered 2 to 5) are used on Cluster, with full scales and the corresponding digital resolutions as shown in Table 1. Another range (range 7) was used only for ground testing.

Switching between ranges is either automatic, controlled by the instrument Data Processing Unit (DPU) in flight, or set by ground command. When in the automatic mode, a range selection algorithm running in the DPU continuously monitors each component of the measured field vector. If any component exceeds a fraction (set at $90 \%$ ) of the range, an up-range command is generated and transmitted to the sensor at the start of a new telemetry format. (All three components are measured in the same range.) If all three components are

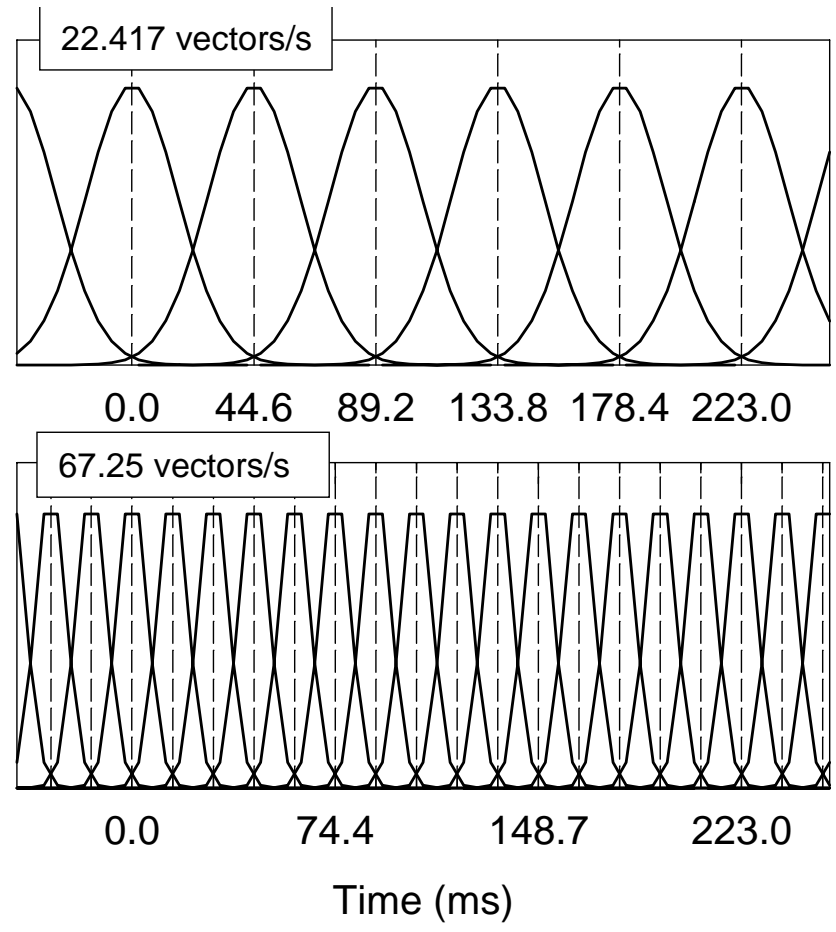

Fig. 2. Gaussian digital filtering of the full resolution sampling at 201.75 vectors/s of the magnetic field. The two most frequently used vector sampling rates are illustrated.

smaller than $12.5 \%$ of the range for more than a complete spin period, a down-range command is implemented at the start of the next vector. The facility to override the automatic ranging is included partly for test purposes, and partly as a capability for failure recovery.

\section{Overview of the FGM instrument}

The sampling of vectors from the magnetometer sensor designated as the primary sensor is carried out at the rate of 201.793 vectors/s. This internal sampling rate has been selected to provide an appropriate set of lower rates (after filtering) for the different telemetry modes and to give the highest frequency response for the short periods of interest recorded in the MSA. In order to ensure the high stability of the sampling rate, the clock signal used for it is derived from a $2^{23} \mathrm{~Hz}$ crystal oscillator internal to the instrument. The primary requirement is that the sampling of vectors be carried out at equal time intervals. This requirement is implemented by sequencing the software by the sampling clock and by ensuring that all software sequences have a deterministic duration. The full bandwidth of the sampled vectors cannot be routinely transmitted via the telemetry due to the limited telemetry rate allocation. The Central Processor Unit encompasses the full bandwidth of data with a Gaussian digital filter to match the rate and bandwidth of the transmitted vectors to the available telemetry rate. The filter coefficients are selected from stored sets corresponding to the different 


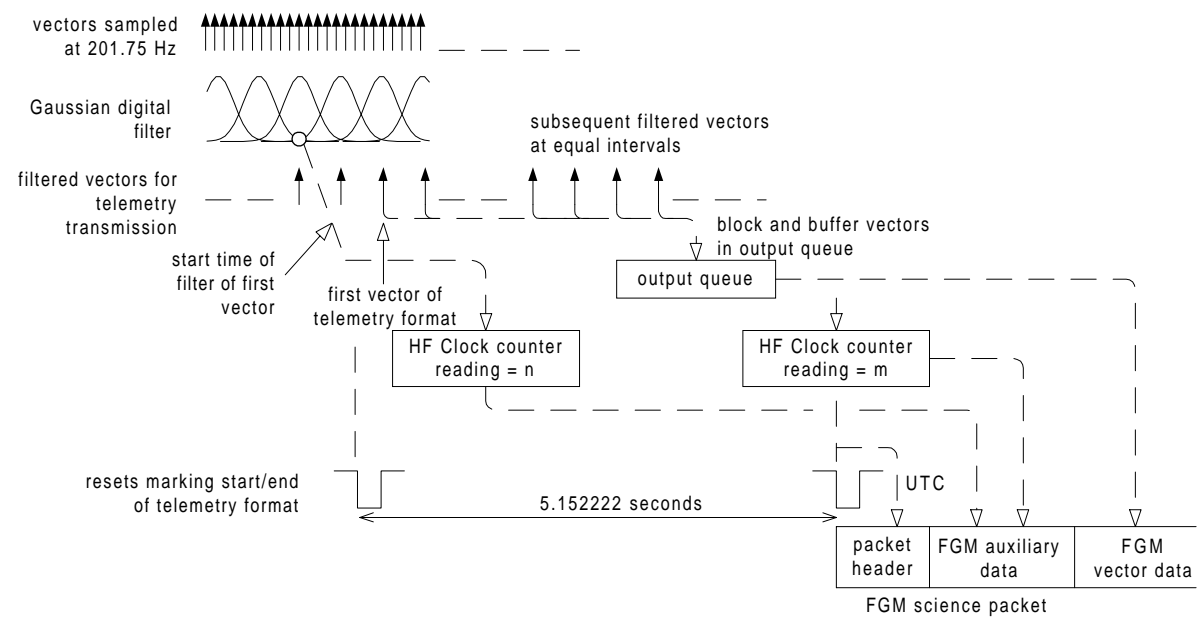

Fig. 3. Internal generation of the timing data for transmission though the spacecraft telemetry to ensure the accurate reconstruction of the vector timing by the ground processing.

telemetry modes. Filtering in the two most frequently used instrument modes is illustrated in Fig. 2.

Following the filtering, completed vector samples are stored in an output queue for the duration of a telemetry frame between reset periods. The recovery of the times at which the vectors were sampled is a very important requirement. The timing chain which enables the conversion (after data recovery on the ground) is indicated schematically in Fig. 3. While timing of the samples is strictly based on the internal FGM clock, by recording and transmitting a time marker, based on the spacecraft provided HF clock, which corresponds to the first vector sample in a given telemetry frame, together with the reading of the same clock at the time of the start of the next telemetry frame, the vector sample sequence can be unambiguously recovered.

\section{Operational modes of the instrument}

The operational modes of the instrument are matched to the different scientific telemetry modes of the spacecraft that define the data rates available to the FGM. Additional parameters of the operational modes are the proportions of data from the primary and secondary sensors, as well as whether data are read out from the Microstructure Analyser memory. The main operational modes of the FGM instrument are summarised in Table 2, together with the associated vector transmission rates through the telemetry.

A particular operation mode implemented late in the Cluster 1 programme and not described before allows one to overcome the limited sampling along the Cluster orbit. It is clear from the Cluster Master Science Plan that there are significant periods when there is no telemetry acquisition, with total data coverage at approximately $50 \%$. It is possible for the FGM instrument to take data during these data gaps and store it within the instrument for later transmission to Earth. To significantly extend the data coverage period, it is neces- sary to reduce the data storage rate to less than that of the normally available FGM modes. A convenient rate is spin synchronised with one averaged vector per spin being stored to memory. In this way, an additional $27 \mathrm{~h}$ of data can be recovered from periods when there is no telemetry coverage. This is the FGM Extended Mode (FGMEXT).

Each FGM contains 192 Kbytes (96 K words) of memory, the Micro Structure Analyzer (MSA) which is normally used for capturing short periods of high resolution data. At the beginning of LOS (Loss of Signal), the FGM is commanded into FGMEXT which uses the MSA to store long periods of spin averaged data. Naturally, this data must be read out as soon as telemetry is restored so that the memory can revert to its normal purpose. This is achieved through the use of the BM3 dump (FGMOPM8) which normally occurs soon after the next acquisition of signal (AOS). FGMEXT was tested during the early phase commissioning of the FGM but did not become fully operational until after the BM3 dumps at AOS were routinely implemented in the Master Science Plan; thus, FGMEXT became operational on orbit 113 in February 2001. Data are taken only by the primary sensor (the outboard sensor by default); the primary sensor remains in the autoranging mode.

In this mode, the instrument synchronises acquisitions to spin sectors by measuring the Sun Reset Pulse (SRP) period and dividing by 512 . These are then despun, averaged and stored to the MSA. Data timing is by the HF clock $(4096 \mathrm{~Hz})$ at points derived from a measure of the spin period. For example, with a $4 \mathrm{~s}$ spin period, each data point will be 32 ticks separated from its neighbours on either side. Data acquisition is performed by calls to the routine normally used to read the secondary vectors and housekeeping voltages. Vectors are acquired with a delay of no more than $100 \mu$ s after the allotted clock transition.

Despinning is achieved in a similar way to that of the Ulysses instrument, i.e. by implementing Walsh transforms with Haar coefficients, where the Haar coefficients are given 
Table 2. Magnetic vectors telemetered to ground for the operating modes of FGM

\begin{tabular}{cccc}
\hline Spacecraft TM modes & $\begin{array}{c}\text { FGM } \\
\text { telemetry modes }\end{array}$ & $\begin{array}{c}\text { Vectors/s } \\
\text { (primary sensor) }\end{array}$ & $\begin{array}{c}\text { Vectors/s } \\
\text { (secondary sensor) }\end{array}$ \\
\hline Nominal Modes 1, 2, 3 & A & 15.519 & 1.091 \\
Burst Mode 2 & B & 18.341 & 6.957 \\
Burst Mode 1 & C & 22.416 & 3.011 \\
Burst Mode 3 & D & 67.249 & 7.759 \\
\hline
\end{tabular}

by the equations in Table 3.

The Haar functions are simply +1 where the corresponding trigonometric function is positive and -1 where the corresponding function is negative. They are undefined at the zero crossing points of the functions. As the number of points per spin is a power of 4 , this means that data points are taken where the functions are undefined. For this reason the Haar functions are given a phase delay so that the transition lies between vectors. This is achieved onboard by establishing what quadrant a particular vector is being calculated in and stating explicitly what the Haar coefficients are for all vectors in that quadrant, i.e. the coefficients are not calculated for each new angle.

The despun components are calculated from the spinning measurements, as shown in the equations in Table 3. Each vector is the arithmetic mean of 512 despun vectors over a single spacecraft spin. The data is susceptible to aliasing as the acquisition frequency is not greater than twice the cut off frequency of the onboard analogue filter, set to $90 \mathrm{~Hz}$. Furthermore, the digital filter is a top hat which has associated side lobes in the frequency domain. Assuming that the spin period is $4 \mathrm{~s}$ and the code acquires a vector on the required $\mathrm{HF}$ clock tick (frequency $=4096 \mathrm{~Hz}$, then each component will be consistent with a resolution of $0.022^{\circ}$.

Data sampled in FGMEXT is also written to the OnBoard Data Handling System (OBDH). Although there is no scientific telemetry, there is continuous housekeeping monitoring. For this reason, the FGM provides a reduced set of safety critical housekeeping parameters (power supply temperature, voltages, and reset count) for the purposes of onboard monitoring.

Once the FGM is switched into FGMEXT, it remains in this mode until the instrument has filled up the MSA $(23 \mathrm{~h}$ initially but this may be ramped up to $28 \mathrm{~h}$ depending on inflight performance) or is switched back to FGMOPM1 due to AOS occurring. In both cases, the contents of the MSA are automatically frozen until transmission to Earth has been completed. It is possible to download the MSA contents in other telemetry modes (e.g. FGMOPM3) but the BM3 option is the most efficient. The housekeeping packet will provide an input into the Data Processing Software module that deals with FGMEXT, together with the contents of the MSA dump in order that the vector timeline can be accurately recovered. The $\mu / 4$ term is also included at this stage.

\section{In-flight calibration: techniques and preliminary re- sults}

The calibration of the magnetic field data is of key importance for meeting the scientific objectives not only of the magnetic field investigation, but also those of the mission as a whole, as the interpretation of observations in terms of physical processes relies on the detailed comparison of measurements made at the four spacecraft. Calibration in this context represents the determination of parameters that allow for the transformation of raw measurements transmitted through the telemetry into a magnetic field vector, given in physical units (nT), in an instrument-specific coordinate system that is unambiguously related to the coordinate system of the spacecraft. The calibration parameters are used by the data processing software (see Sect. 5) to generate the magnetometer data in a range of geophysical coordinate systems.

The in-flight calibration of FGM is based on an evaluation of all the possible sources of errors that occur in the measurement process, embodied in an "instrument model" representing the measurement process of the magnetic field. Conceptually, given the actual value $\boldsymbol{B}_{\mathrm{GSE}}$ of the ambient magnetic field vector at the location of the FGM sensor (given, for instance, in Geocentric Solar-Ecliptic, GSE, coordinates), the FGM output through the telemetry is a digitised vector $\boldsymbol{V}$. This vector (the actual measurement) depends on in a complex way the alignment and orthogonality of the sensor axes with respect to the GSE coordinate system. This depends on the scale factors and offsets of the sensors and electronics of FGM and on the offsets introduced by the spacecraft. The instrument model also needs to take into account the time and frequency response in the form of delays and effective bandwidth due to the magnetometers, the Analogue-to-Digital Converters, and the digital filtering process. The coordinate transformation from GSE into the (nearly, but not quite orthogonal) magnetometer sensor system (specific to each of the eight magnetometers on the four Cluster spacecraft) is a complex superposition of transformations that also takes into account the misalignements introduced by the spacecraft, the magnetometer booms, sensor mounting and construction. All these effects need, in principle, to be evaluated for each of the measured output vectors.

Ground calibration of the FGM instruments has allowed for the development of a practical model of the instruments, identifying the effective transformations that lead from the 
Table 3. HAAR function definition for the extended mode use of the MSA

\begin{tabular}{ll}
\hline$C_{H}(t)=\frac{\operatorname{Cos}(\omega t)}{|\operatorname{Cos}(\omega t)|}$ & $S_{H}(t)=\frac{\operatorname{Sin}(\omega t)}{|\operatorname{Sin}(\omega t)|}$ \\
\hline$B_{Y}=\frac{\sum_{i=0}^{N-1} \frac{\pi}{4}\left[-B_{Z S i} S_{H i}+B_{Y S i} C_{H i}\right]}{N}$ & $B_{Z}=\frac{\sum_{i=0}^{N-1} \frac{\pi}{4}\left[B_{Z S i} C_{H i}+B_{Y S i} S_{H i}\right]}{N}$ \\
$B_{Z}=$ Despun $Z$ component & $B_{Z S}=\operatorname{Sampled} Z$ component \\
$B_{Y}=$ Despun $Y$ component & $B_{Y S}=$ Sampled $Y$ component \\
$N=$ Number of points per spin $=512$ & $C_{H}, S_{H}=$ Haar functions \\
\hline
\end{tabular}

ambient field to the measured output in the following form:

$\boldsymbol{V}=\mathbf{c}^{(\text {instr })} \mathbf{c}^{(S R-F S R)} \mathbf{c}^{(\text {spin })} \mathbf{c}^{(\text {att })} \boldsymbol{B}_{\mathrm{GSE}}+\boldsymbol{c}_{0}$.

The sensor matrix $\mathbf{c}^{(\text {sensor })}=\mathbf{c}^{(\text {instr })} \mathbf{c}^{(S R-F S R)}$ represents the sensitivities (scale factors) of the sensors and the alignment of the three sensor axes with respect to an orthogonal coordinate system aligned with the real spin axis. The spacecraft spin is taken into account in the rotation matrix $\mathbf{c}^{(\text {spin })}$, corresponding to the spin-phase angle of sensors at the time of the measurement. The matrix $\mathbf{c}^{(a t t)}$ represents the transformation into the spacecraft spin-aligned coordinate system from the GSE system. This matrix is determined from the spacecraft attitude measurements. Finally, the vector $\boldsymbol{c}_{0}$ represents the offsets associated with the sensors and the spacecraft background field at the location of the sensors.

The equation defining the measurements can be transformed to state the in-flight calibration task, i.e. to determine the relevant term in the inverted form of this equation:

$\boldsymbol{B}_{\mathrm{GSE}}=\mathbf{c}^{(a t t)^{-1}} \mathbf{c}^{(\text {spin })^{-1}} \mathbf{c}^{(S R-F S R)^{-1}} \mathbf{c}^{(i n s t r)^{1}}\left(\boldsymbol{V}-\boldsymbol{c}_{0}\right)$.

In the following, the steps performed by the first modules of the data processing software are described that implement the practical definition of the calibration parameters that need to be derived from the data. The digital output of the magnetometer is a vector $\boldsymbol{V}$ in binary units. As a preparatory step, this vector is transformed into physical units, using a diagonal matrix of nominal scale factors $\mathbf{M}_{S T}$ (nT/binary count), to a vector $\boldsymbol{B}_{F S}$ corresponding to the tri-axial magnetometer output in magnetic field units (uncalibrated, or "raw" nT):

$\boldsymbol{B}_{F S}=\mathbf{M}_{(S T)} \boldsymbol{V}$

The coordinate system used in this equation is the FGM sensor system, defined by the true (magnetic) directions of the sensor triad; it is not exactly orthogonal, due to small inaccuracies in the magnetic and mechanical alignment of the sensors. The vector $\boldsymbol{B}_{F S}$ is used as an input to the next processing step which then uses the calibration parameters to yield the magnetic field vector in physical units. In a coordinate system, this is denoted by FSR, which is the FGM spin reference system, an orthogonal, right-handed coordinate system with its $X$-axis along the real spin axis of the spacecraft, and the $Y$-axis aligned with the sensor along the magnetometer boom. The defining equation of this transformation is

$\boldsymbol{B}_{F S R}=\mathbf{c}^{(c a l)} \boldsymbol{B}_{F S}-\boldsymbol{o}^{c a l}$.

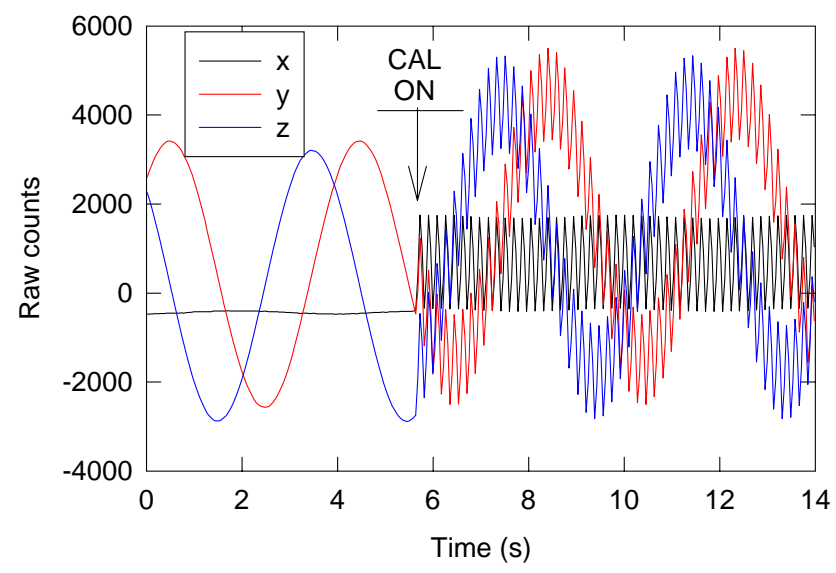

Fig. 4. Implementation of the in-flight calibration mode of the FGM instrument. The data illustrated are from an actual calibration sequence on spacecraft 1 . When the calibration mode is switched on, a train of square wave, consisting of 512 on-off applications of a fixed value calibration signal is internally superposed on the ambient field measured by the instrument. The frequency of the square wave is matched to the filtering bandwidth of the vector sampling.

The objective of the in-flight calibration is to determine the $3 \times 3$ matrix $\mathbf{c}^{(\mathrm{cal})}$ and the offset vector $\boldsymbol{o}^{\mathrm{cal}}$ used in this equation for all magnetometers on the four spacecraft, for all ranges and, in each case, for both A-D converters. The calibration matrix $\mathbf{c}^{(\mathrm{cal})}$ depends on a number of effects that include range-dependent scale factors, deviations of the sensor axes from orthogonality, scale factor deviations between the two A-D converters and the alignment of the sensor axes to the spin axis system. The offset vector $\boldsymbol{o}^{\text {cal }}$ depends on both spacecraft-induced and sensor offsets. Despinning, i.e. the application of the matrix $\mathbf{c}^{(\text {spin })^{-1}}$, as well as the coordinate transformation $\mathbf{c}^{(a t t)^{-1}}$ into a physical coordinate system, such as GSE, are performed, as outlined below, in the routine data processing of the FGM data. The built-in inflight calibration mode (FGMCAL) is initiated nominally once per orbit, or at a slower rate, as considered appropriate, given that its application involves the disabling of the FGM data on Inter-Experiment Link, preventing other investigator from receiving the data used for their onboard processing. This mode provides information on the sensitivity factors and alignment of the three axes of the FGM primary sensor. In this mode, an internally generated calibration signal is applied to the primary sensor, following a preset sequence. The full sequence consists of 512 cycles of CAL ON and CAL OFF steps, with a $50 \%$ duty cycle. The frequency of the steps is one half of the Nyquist frequency of the current primary science output. For instance, in the nominal mode (FGM Op Mode 1), at 22.4217 vectors/s transmission rate, the frequency of the on-off calibration signal is $22.4217 / 4=5.60 \mathrm{~Hz}$. The operation of the in-flight calibration mode is illustrated in Fig. 4. The raw telemetry output of the instrument is illustrated, prior to despinning, calibration and transformation into physical units and phys- 


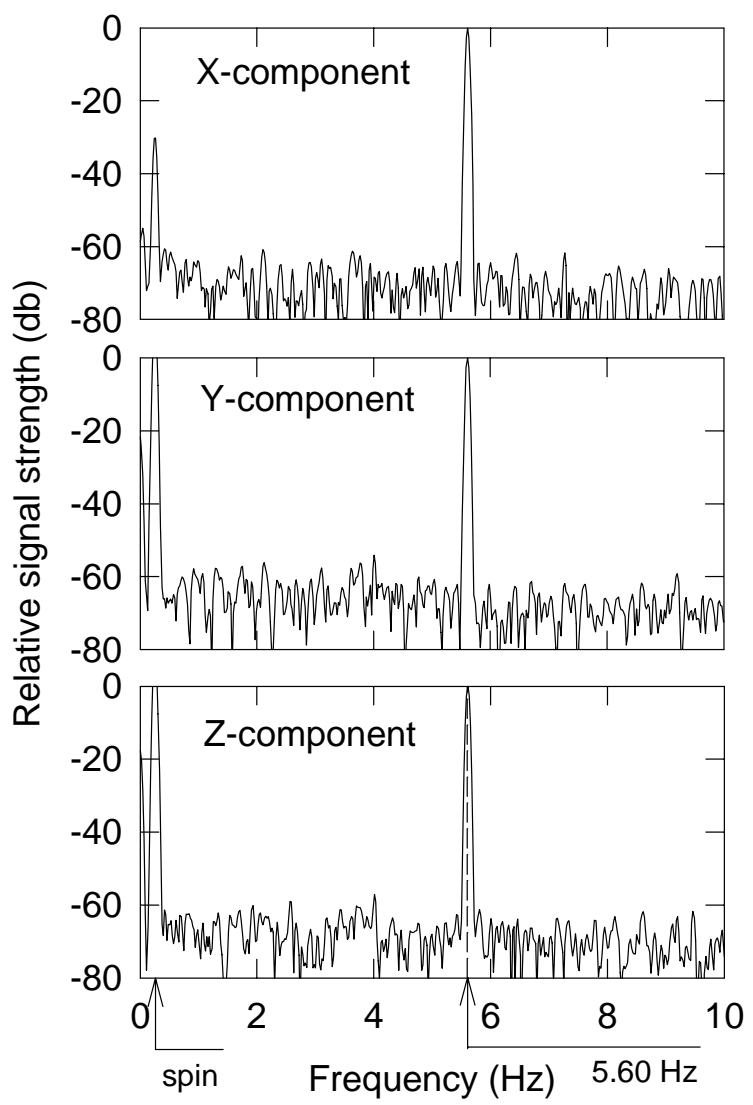

Fig. 5. Frequency domain analysis of the in-flight calibration sequence shown in Fig. 4 for the three components of the magnetic field. The lower two panels show the spin plane components, the upper panel shows the nominally spin-aligned component. The signal-to-noise ratio of the calibration signal (at $5.90 \mathrm{~Hz}$ ) is more than $60 \mathrm{db}$.

ical coordinates. The calibration signal is superimposed on the measured components of the background (external) magnetic field that is being measured. The $Y$ - and $Z$-components of the magnetic field are in the spinning coordinate system of the magnetometer sensor, while the $X$-component of the field is taken along the spin axis. The routine analysis of the calibration data, carried out on the ground, is illustrated in Fig. 5. The analysis software essentially carries out a Fast Fourier Transform (FFT) to measure the response of the sensors and associated electronics to the imposed magnitude of the calibration step. Due to the periodic application of the step, its magnitude can be measured, as is evident in Fig. 5, with a very high (better than $60 \mathrm{db}$ ) signal-to-noise ratio. A routine monitoring of the response of the sensors (on all four spacecraft) provides a test of the long-term stability of the magnetometers. The FFT analysis of the calibration data also provides a measure of the signal at the spin frequency for all three instrument axes. For the spinning $(Y$ and $Z)$ components, the ambient field generates a signal with an amplitude equal to the component of the magnetic field in the spin plane, whereas for the nominally spin aligned sensor axis $(X)$, the signal at the spin frequency represents the mis-

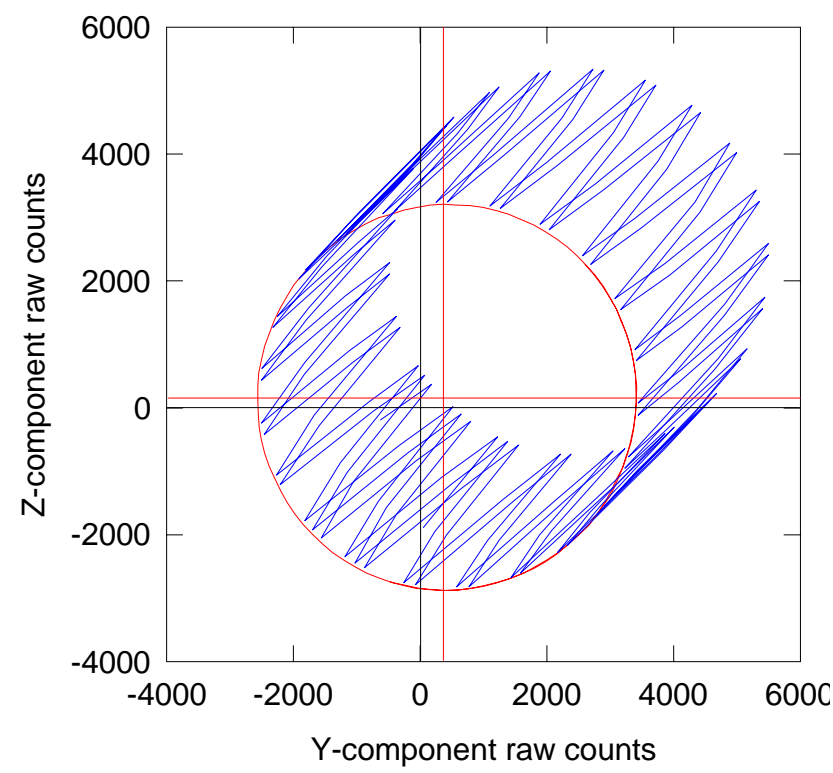

Fig. 6. Plot of the two spin plane components of the (raw) magnetic field data prior to and during the application of the calibration signal (in blue). The circle (in red) represents the ambient magnetic field measured by the two sensor axes; the offset of the circle from the origin represents the offsets of these two axes.

alignment (or "coning") of the sensor axis with respect to the spacecraft spin. Given that in this instance, the spinning components have a (relative) magnitude of $8.2 \mathrm{db}$ and the spin signal in the $X$-component is $30.2 \mathrm{db}$, the ratio of the spinning component along the $X$-axis to the spin plane component is 0.012 , corresponding to a $0.69^{\circ}$ end-to-end deviation of the sensor from the spin axis, a value well matched by other determinations of the sensor alignment. A plot of the spinning components ( $Z$-component vs. $Y$-component) is illustrated in Fig. 6 for the calibration period shown in Fig. 4. Prior to the application of the calibration signal, the spinning components describe a circle, corresponding to the ambient field. The centre of the circle gives the offset values for the spinning components.

In addition to analysing the calibration mode data, there are a number of statistically based analysis techniques that use variations in the physical measurements to determine a number of calibration parameters. Figure 7 illustrates, in a simplified way, the corrections made during the calibration. Small corrections to the sensitivities of each sensor affect the scaled value of the field component to $\mathrm{nT}$; small misalignments from the spin axis of the spacecraft and deviations from the orthogonality mix contributions from the measured components, and offsets or zero levels effect the absolute field values. If the effects are linear in these parameters (for example, they do not depend on the measured field), errors in these quantities introduce anomalous power at the spin frequency and harmonic, when the data are despun (transformed into a fixed coordinate system). The basic calibration analysis technique seeks to find certain combinations of parame- 

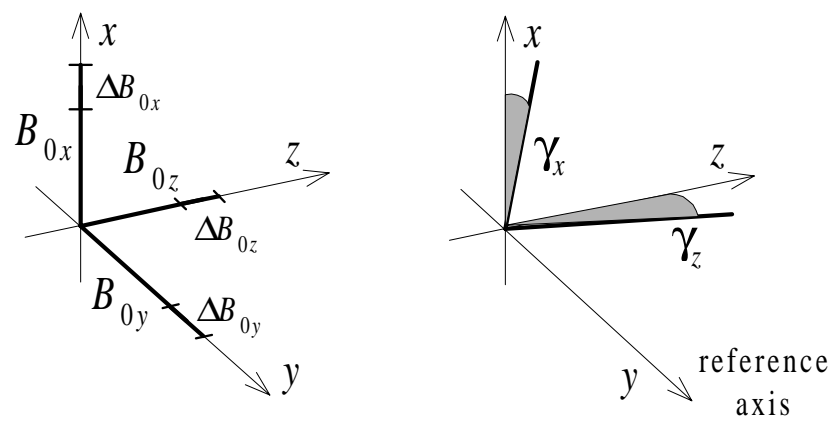

Fig. 7. Schematic representation of the offset and alignment of the FGM sensor axes. Values of the offsets and the measured values of the misalignment are given in Table 4.

ters (such as offsets, gain ratios, misalignments and orthogonality angles) by removing (to background) these anomalous power bands (spin tones). A direct method to perform this in a linear based system is singular value decomposition. Only certain parameters separate in this analysis, so that other independent methods may be applied, or assumptions about the character of the data are sometimes made at intervals. The spin axis offset, for example, does not contribute directly to the spin tones, and requires special techniques, such as the Hedgecock technique in the solar wind to check its value. On Cluster, there is also the possibility of combining the measurements of the magnitude of the magnetic field by the Electron Drift Instrument (EDI) to estimate corrections to the spin axis offset. This method has been previously used successfully on the Equator-S mission (Fornacon et al., 1999).

The four spacecraft system also provides an opportunity to proceed beyond the single spacecraft analysis and check the absolute orientation of the spin axes of the spacecraft. Other less well determined parameters can also be checked in principle, such as the spin axis gains. The four-point calibration of the magnetic field vector measurements and the evaluation of the magnetic field gradients were specifically addressed by Kepko et al. (1996), Khurana et al. (1996) and Khurana et al. (1998).

A combination of these calibration techniques has yielded a good absolute calibration of the magnetometers on all four Cluster spacecraft during the Commissioning Phase of the instruments. A summary of the representative calibration values is given in Table 4. Calibration files (corresponding to the calibration matrix $\mathbf{c}^{c a l}$ and the offset vector $\boldsymbol{o}^{\text {cal }}$ ) are routinely provided for processing the data from all four spacecraft. In the first instance, calibration files are generated as daily files, based on quick-look data downloaded from the Cluster Data Disposition System in ESOC. These files are normally used for processing spin-averaged ( $4 \mathrm{~s}$ resolution) vectors. High time resolution magnetometer data, used for the application of special four-point analysis tools (for instance, the discontinuity analyser for boundary determinations, the curlometer for calculating the current density vector and the wave telescope for investigating wave modes and wave vectors) usu-

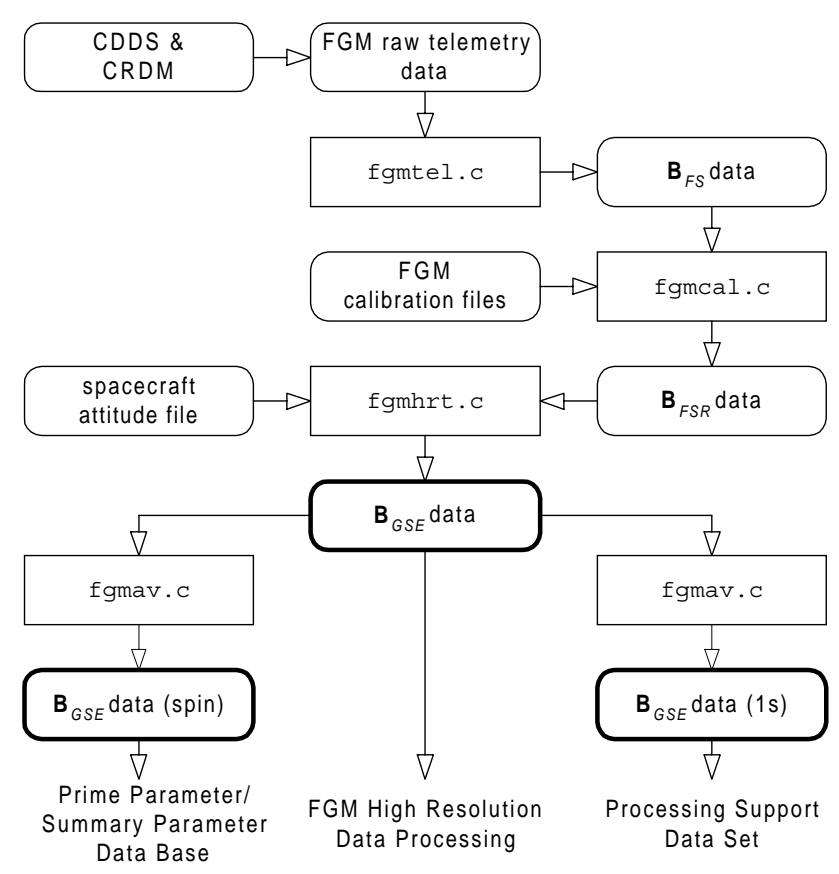

Fig. 8. Simplified flow diagram of the FGM data processing software.

ally require additional calibration.

\section{Data processing: summary of data throughput and data products}

The data processing software routinely applied to the FGM data has the following main tasks:

- Transformation of the raw telemetry data into a format suitable for further processing;

- Reconstruction of the time at which the vector data were measured;

- Application of the calibration parameters to correct for instrumental and other effects in the data in order to recover the accurate value of the magnetic field at the location of the sensors;

- Transformation of the magnetic field vectors into standard geophysical coordinate systems.

The data processing also performs the following additional tasks:

- Merging of the spacecraft position vectors to the magnetometer data streams;

- Averaging the measured magnetic field vectors over different time intervals;

- Providing appropriate data interfaces for the generation of standard FGM data products. 
Table 4. Calibration summary for the outboard sensor

\begin{tabular}{ccccc}
\hline & FGM SC1 & FGM SC2 & FGM SC3 & FGM SC4 \\
\hline$B_{0 x}$ & $-2.5 \mathrm{nT}$ & $0.0 \mathrm{nT}$ & $-1.5 \mathrm{nT}$ & $-14.0 \mathrm{nT}$ \\
$B_{0 y}$ & $4.0 \mathrm{nT}$ & $-2.0 \mathrm{nT}$ & $-5.0 \mathrm{nT}$ & $-3.5 \mathrm{nT}$ \\
$B_{0 z}$ & $1.0 \mathrm{nT}$ & $-0.5 \mathrm{nT}$ & $-3.0 \mathrm{nT}$ & $4.5 \mathrm{nT}$ \\
$\Delta B_{0 x}, \Delta B_{0 y}, \Delta B_{0 z}$ & \multicolumn{5}{c}{$<0.2 \mathrm{nT}$} \\
$\gamma x$ & $0.7^{\circ}$ & $0.4^{\circ}$ & $0.8^{\circ}$ & $0.4^{\circ}$ \\
$\gamma z$ & $0.6^{\circ}$ & $0.7^{\circ}$ & $0.7^{\circ}$ & $1.2^{\circ}$ \\
Scale factors errors & \multicolumn{5}{c}{$\sim 0.1 \%$} \\
\hline
\end{tabular}

A simplified flow diagram of the FGM data processing software structure is shown in Fig. 8. The basic input to the processing comes from either the Cluster Data Disposition System (CDDS) at ESOC, representing quick-look data, or from the Cluster Raw Data Medium (CRDM), the CDROMs used for the distribution of Cluster data. The modular nature of the software is apparent in Fig. 8; the different modules implement the transformations enumerated and described above. The module fgmtel. $\mathrm{c}$ unpacks the telemetry and generates vectors in physical units in the (unorthogonalised) sensor coordinate system. This module also generates the timing and spin phase information for each measured vector. The module fgmcal. $\mathrm{c}$ incorporates the calibration files, determined outside the processing chain, and generates the orthogonalised vectors in the spin-aligned coordinate system. The following software module, fgmhrt.c, despins the vectors, and, using the spacecraft attitude data, yields a time series in a selected geophysical system, normally in GSE, at the highest resolution in the current mode of the instrument, according to $\boldsymbol{B}_{\mathrm{GSE}}$ using the notation from the previous section of this paper. This is the form of the FGM software used to

$\boldsymbol{B}_{\mathrm{GSE}}=\mathbf{c}_{(a u t)^{-1}} \boldsymbol{B}_{F S R}$

generate magnetic field data within the investigator team. Additional features (processing of the spacecraft position and averaging, using the fgmav. c module) are used as appropriate.

The FGM magnetic field data are widely used by the Cluster investigator community, both to process their own data and for cooperative studies with the magnetometer team. Similarly, FGM data are also used in connection with ground-based investigations. The provision and distribution of magnetic field data represents a major effort undertaken by the FGM team to support the exploitation of the Cluster mission as a whole.

FGM data are routinely processed, using software supplied by the FGM team in the UK Cluster Data Handling Facility (UK-CDHF), a part of the Cluster Science Data System (CSDS), to generate the spin-averaged ( $\sim 4 \mathrm{~s}$ resolution) Prime Parameter (PP) vector data from all four Cluster spacecraft, as well as the Summary Parameter (SP) vector data from one spacecraft at a $1 \mathrm{~min}$ resolution. These data are

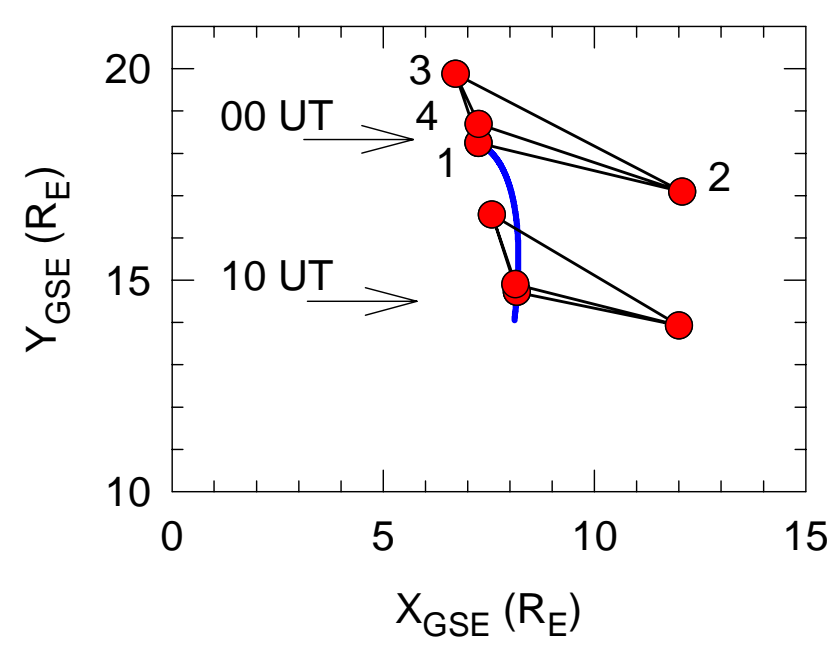

Fig. 9. Representation of the Cluster constellation in the $X-Y$ plane in the GSE coordinate system on 25 December 2000. The spacecraft constellation is shown magnified by a factor of 30 .

then distributed through the CSDS to the other Cluster Data Centres, and to the Cluster scientific community. These data form the basis for most of the collaborative studies with the FGM team.

In addition, a number of Cluster investigations require FGM data at a higher $1 \mathrm{~s}$ resolution, for generating their own Prime Parameter data. A particular implementation of the FGM processing software has been made widely available to both CSDS Data Centres and to Cluster investigators, to generate the so-called Processing Support Data Set (PSDS). These data are used routinely for processing the data from other Cluster investigations, such as PEACE, CIS, EDI and EFW.

\section{Overview of the early phase observations}

From its first commissioning operations in August 2000, Cluster and the FGM instruments on the four spacecraft demonstrated that the four-point measurements are able to bring a new understanding of magnetospheric phenomena. Due to the extended commissioning phase of the mission and all its instruments, routine four-spacecraft magnetic field measurements have only been available from December 2000; once such measurements became available, it also became possible to address the scientific objectives of the mission. In the following, we present brief examples of magnetic field measurements, demonstrating the capabilities and performance of the instrument. An early cusp crossing in August 2000 with simultaneous magnetic field observations was observed near perigee by three Cluster spacecraft (the fourth instrument had not yet been switched on). Routine observations of the outer cusp have been performed by Cluster since January 2001 (see Cargill et al., 2001). For initial bow shock studies, the sequence of crossings on 25 December 2000 is particularly suitable. This series of shock cross- 

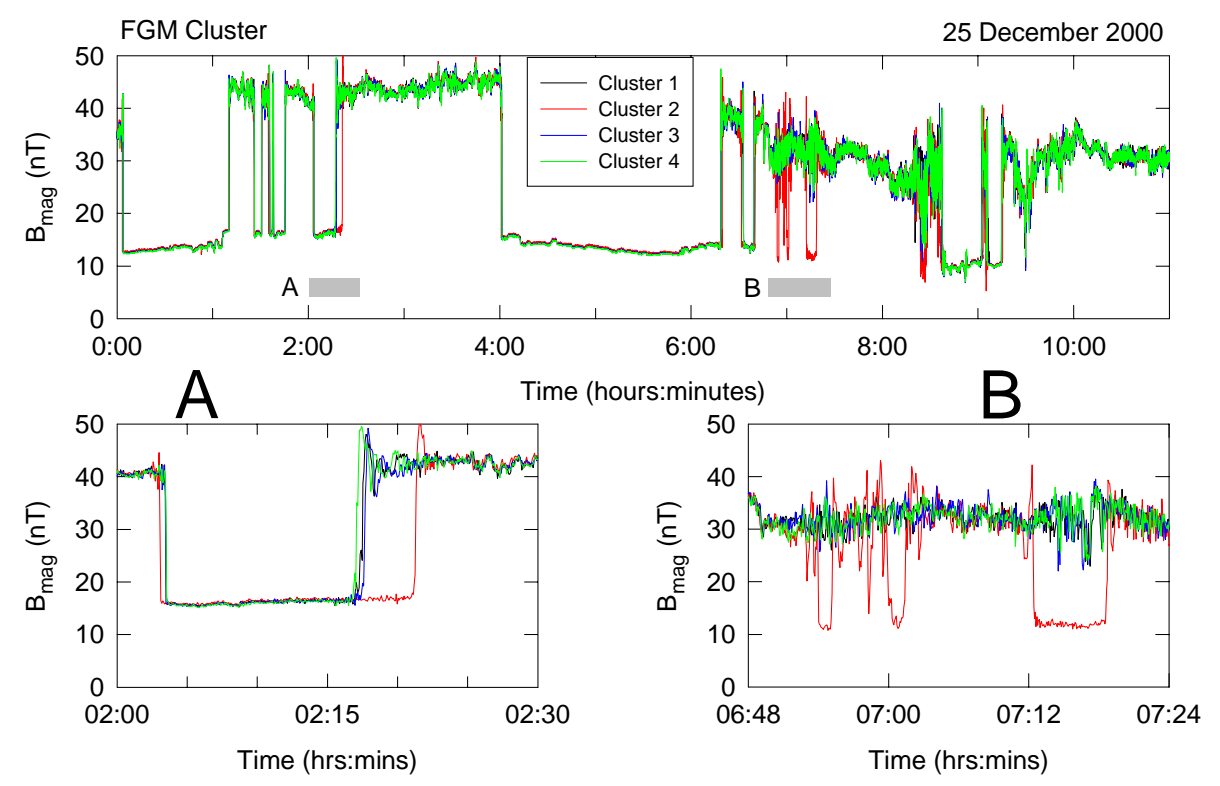

Fig. 10. An overview of the multiple crossings of the bow shock by Cluster on 25 December 2000, with two intervals shown in higher resolution to illustrate the motion and dynamics of the bow shock observed by Cluster.

ings is examined in more detail by Horbury et al. (2001). The orientation of the constellation was, at this time (near apogee), such that spacecraft 1,3 and 4 were close to a plane perpendicular to the Earth-Sun line, while spacecraft 2 was sunward of the other three, between about 1100 and $1400 \mathrm{~km}$, as shown in Fig. 9. Although during this period of the mission, the plasma instruments on Cluster remained switched off, it is possible to examine, in considerable detail, the variety of shock phenomena observed in these relatively low Mach number shocks for which the plasma beta parameter (representing the ratio of the upstream plasma pressure to the magnetic field pressure) remained low, and the solar wind magnetic field remained relatively high $(>12 \mathrm{nT})$. An interesting feature of these shocks is that in spite of their appearance as classical perpendicular bow shock waves when viewed at a resolution of $4 \mathrm{~s}$, there is considerable structure associated with them, as seen at high resolution, which represents fast dynamic processes, on time scales considerably shorter than their overall motion (Horbury et al., 2001). Figure 10 illustrates the large-scale dynamics of the shock, as seen by the magnetometers on the four spacecraft.

Acknowledgements. The Cluster mission and the FGM team are greatly indebted to many individuals who made the mission possible and who contributed greatly to its success, as well as to the success of the FGM instrument. The rebirth of the mission, from the disaster of 1996, is due in the largest measure to the determination of Prof. Roger Bonnet, ESA's Director of Scientific Programmes and of John Credland, ESA's Head of Scientific Projects and previously Cluster Project Manager. We thank them also for partly funding the rebuilding of the FGM instruments. The Project Teams at ESA (in particular John Ellwood, Bodo Gramkow, Joseph Pereira, Colin Parkinson) and at Dornier, now Astrium (in particular Günther Lehn, Roland Nord, Erwin Kraft) have greatly contributed to the successful rebuilding of the Cluster spacecraft and their preparation for launch. We also remember the considerable contribution to Cluster by Gus Mencke and the late Hans Bachmann. We are grateful to STARSEM for the successful and spectacular launches and to the Cluster Flight Operations Team at ESOC (in particular to Manfred Warhaut, Sandro Matussi and Colette Pullig) for their ready support of FGM operations. The support of the Cluster Project Scientists, first of Rudi Schmidt, later of Philippe Escoubet and Michael Faehringer is gratefully acknowledged. The rebuilt FGM instrument is very close to the original design developed by the earlier Technical Managers, Ray Carvell and John Thomlinson; the flight software still bears the imprint of its original designer, Ed Serpell. Also contributing to the hardware and calibration of the FGM were R. Kempen, H. Kügler, M. Rahm, I. Richter at the Technische Universität Braunschweig; J. Scheifele at the Goddard Space Flight Center; O. Aydogar at the Institut für Weltraumforschung, Graz. Special thanks are due to Mrs S. Balogh, Imperial College, for administrative and project support.

Financial support for the Cluster magnetic field investigation has been provided, in addition to ESA, by the Particle Physics and Astronomy Research Council in the United Kingdom (our special thanks to Mrs Sue Horne), DLR in Germany, the Österreichische Akademie der Wissenschaften in Austria and NASA in the United States (with special thanks to Dino Macchi).

\section{References}

Balogh, A., Cowley, S. W. H., Dunlop M. W., et al.: The Cluster magnetic field investigation: Scientific objectives and instrumentation, in Cluster: mission, payload and supporting activities, ESA SP-1159, 95, 1993.

Balogh, A., Dunlop, M. W., Cowley, S. W. H., Southwood, D. J., Thomlinson, J. G., and the Cluster magnetometer team: The Cluster magnetic field investigation, Space Sci. Rev., 79, 65, 1997. 
Balogh, A. and Dunlop, M. W.: FGM-specific multi-point analysis, Proc. Cluster II Workshop on Multiscale/Multipoint Plasma Measurements, ESA SP-449, 147-154, 2000.

Cargill, P. J., Dunlop, M. W., Balogh, A., and the FGM team: First Cluster results of the magnetic field structure of the mid- and high-altitude cusps, Ann. Geophysicae, (this issue) 2001.

Dunlop, M. W., Woodward, T. I., Motschmann, U., Southwood, D. J., and Balogh, A.: Analysis of non-planar structures with multipoint measurements, Adv. Space Res., 18, 8, 309-314, 1996.

Dunlop, M. W., Woodward, T. I., Southwood, D. J., Glassmeier, K.H., and Elphic, R. C.: Merging 4 spacecraft data: Concepts used for analysing discontinuities, Adv. Space Res., 644, 1101-1106, 1997.

Dunlop, M. W. and Woodward, T. I.: "Discontinuity analysis: orientation and motion", in: "Analysis Methods for Multispacecraft Data”, ISSI Science Report, SR-001, Kluwer Academic Publisher, 1998.

Dunlop, M. W. and Woodward, T. I.: Cluster magnetic field analysis techniques, Proc. Cluster II Workshop on Multiscale/Multipoint Plasma Measurements, ESA SP-449, 351-354, 2000.

Dunlop, M. W., Balogh, A., Cargill P. J., and the FGM team: Cluster observes the Earth's magnetopause: coordinated four-point magnetic field measurements, Ann. Geophysicae, (this issue) 2001a.

Dunlop, M. W., Balogh, A., Glassmeier K.-H., and the FGM team: Four-point application of magnetic field analysis tools: the curlometer and discontinuity analyser, Ann. Geophysicae, (this issue) $2001 \mathrm{~b}$

Elphic, R. C.: Multipoint observations of the magnetopause: Results from ISEE and AMPTE, Adv. Space Res., 8, 9, 223-238, 1988.

Fornacon, K.-H., Auster, H. U., Georgescu, E., Baumjohann, W., Glassmeier, K.-H., Haerendel, G., Rustenbach, J., and Dunlop, M. W.: The magnetic field experiment onboard Equator-S and its scientific possibilities, Ann. Geophysicae, 17, 1521-1527, 1999.

Giacalone, J., Schwartz, S. J., and Burgess, D.: Artificial spacecraft in hybrid simulations of the quasi-parallel Earth's bow shock: analysis of time series versus spatial profiles and a separation strategy for Cluster, Ann. Geophysicae, 12, 591-601, 1994.

Glassmeier, K.-H., Motschmann, U., Dunlop, M. W., Balogh, A., Acuña, M. H., Buchert, S., Carr, C. M., Fornacon, K.-H., Georgescu, E., Musmann, G., Schweda, K., and Vogt, J.: Cluster as a wave telescope: first results, Ann. Geophysicae, (this issue) 2001.

Horbury, T. S., Balogh, A., Lucek, E. A., Dunlop, M. W., and Cargill, P. J.: Cluster magnetic field observations of the bowshock: motion, orientation and structure, Ann. Geophysicae, (this issue), 2001.

Kepko, E. L., Khurana, K. K., and Kivelson, M. G.: Accurate determination of magnetic field gradients from four point vector mea- surements: 1. Use of natural constraints on vector data obtained from a single spinning spacecraft, IEEE Transact. Magnetics, 32, 377, 1996.

Khurana, K. K., Kepko, E. L., Kivelson, M. G., and Elphic, R. C.: Accurate determination of magnetic field gradients from four point measurements: 2 . Use of natural constraints on vector data obtained from four spinning spacecraft, IEEE Transact. Magnetics, 32, 5193, 1996

Khurana, K. K., Kepko, E. L., and Kivelson, M. G.: Measuring magnetic field gradients from four-point measurements in space, AGU Monograph on Measurement Techniques, 1998.

Lucek, E. A., Dunlop, M. W., Horbury, T. S., Balogh, A., Cargill, P. J., Fornacon, K.-H., Oddy, T., and the FGM team: Cluster magnetic field observations in the magnetosheath: four-point measurements of mirror structures, Ann. Geophysicae, (this issue), 2001.

Paschmann, G.: Magnetopause, cusp and dayside boundary layer: Experimental point of view, Proc. Cluster Workshop on Physical Measurements and Mission Oriented Theory, ESA SP-371, 149$151,1995$.

Paschmann, G. and Daly, P.: Analysis Methods for Multispacecraft Data, ISSI Science Report, SR-001, (Eds), Kluwer Academic Publisher, 1998.

Russell, C. T.: Multipoint measurements of upstream waves, Adv. Space Res., 8, 9, 147-156, 1988.

Rezeau, L. Sahraoui, F., d'Humières, E., Belmont, G., CornilleauWehrlin, N., Mellul, L., Balogh, A., Robert, P., Décréau, P., and Canu, P.: A case study of low-frequency waves at the magnetopause, Ann. Geophysicae, (this issue) 2001.

Scholer, M.: Magnetopause, cusp and dayside boundary layer: Theoretical Point of view, Proc. Cluster Workshop on Physical Measurements and Mission Oriented Theory, ESA SP-371, 153-158, 1995.

Scholer, M. and Burgess, D.: The role of upstream waves in supercritical quasiparallel shock re-formation, J. Geophys. Res., 97, 8319-8326, 1992.

Southwood, D. J.: Multispacecraft measurements at varying scalelengths - the Cluster/Regatta opportunity, Proc. Int. Workshop on Space Plasma Physics Investigations by Cluster and Regatta, ESA SP-306, 1-5, 1990.

Thomsen, M. F.: Multi-spacecraft observations of collisionless shocks, Adv. Space Res., 8, 9, 157-166, 1988.

Wild, J. A., Cowley, S. W. H., Davies, J. A., Khan, H., Lester, M., Milan, S. E., Provan, G., Yeoman, T. K., Balogh, A., Dunlop, M. W., Fornacon, K.-H., and Georgescu, E.: First simultaneous observations of flux transfer events at the high-latitude magnetopause by the Cluster spacecraft and pulsed radar signatures in the conjugate ionosphere by the CUTLASS and EISCAT radars, Ann. Geophysicae, (this issue), 2001. 